\title{
Editorial
}

\section{Intelligent Modeling and Verification 2014}

\author{
Guiming Luo, ${ }^{1}$ Xiaoyu Song, ${ }^{2}$ Xiaojing Yang, ${ }^{3}$ and Krishnaiyan Thulasiraman ${ }^{4}$ \\ ${ }^{1}$ School of Software, Tsinghua University, Beijing 100084, China \\ ${ }^{2}$ Maseeh College of Electrical and Computer Engineering, Portland State University, P.O. Box 751, Portland, OR 97207, USA \\ ${ }^{3}$ Department of Mathematical Sciences, Tsinghua University, Beijing 100084, China \\ ${ }^{4}$ School of Computer Science, University of Oklahoma, 200 Felgar Street, Room 114, Norman, OK 73019, USA
}

Correspondence should be addressed to Guiming Luo; gluo@tsinghua.edu.cn

Received 24 September 2014; Accepted 24 September 2014; Published 22 December 2014

Copyright (C) 2014 Guiming Luo et al. This is an open access article distributed under the Creative Commons Attribution License, which permits unrestricted use, distribution, and reproduction in any medium, provided the original work is properly cited.

After the success of the previous special issue Intelligent Modeling and Verification, which was published last year, we are pleased to announce that the new special issue Intelligent Modeling and Verification 2014 is finally here. Through an extensive peer-review process, 32 papers were selected for publication in the new special issue.

A total of 16 papers describe optimal system modeling. They focus on system modeling, optimal filtering and scheduling, image modeling, pattern recognition, and network modeling.

Based on the total variation model, the paper entitled "Total variation based perceptual image quality assessment modeling" by $\mathrm{Y}$. Wu et al. proposes a new way to assess perceptual image quality in spatial domains. A cluster for use in the film industry is constructed in the paper entitled "Precomputed clustering for movie recommendation system in real time" (B. Li et al.) by implementing a distance matrix based on machine learning techniques. The paper entitled "Unified mathematical framework for slicing and symmetry reduction over event structures" by X. Gao et al. introduces sliced and symmetric quotient reduction models of event structures and presents some corresponding algorithms. Based on the consideration of sharing and real number matrix encoding, the correlation models of virtual resources and the genetic algorithm are presented in "Multitask oriented virtual resource integration and optimal scheduling in cloud manufacturing" (Z. Cheng et al.). The paper entitled "Optimal spatial matrix filter design for array signal preprocessing" by $\mathrm{H}$. Zhang et al. illustrates a technique for designing a spatial matrix filter based on convex programming. In the paper entitled "Constitutive relation of engineering material based on SIR model and HAM" by $\mathrm{H}$. He et al., a dynamic stress-strain model is established according to the homotopy analysis method, and an analytical solution is presented. The paper entitled "The application of pattern recognition in electrofacies analysis" by $\mathrm{H}$. Li et al. introduces the KMRIC algorithm and AKM algorithm. Combining the multigranulations rough set with evidence theory, M. Yan constructs the multigranulations method of the belief and plausibility reductions in the paper entitled "Multigranulations rough set method of attribute reduction in information systems based on evidence theory." Complicated data modeling and adaptive filtering are the main areas of focus for the papers "A new subband adaptive filtering algorithm for sparse system identification with impulsive noise" (Y.-S. Choi ), "A nonlinear multiparameters temperature error modeling and compensation of POS applied in airborne remote sensing system" (J. Li et al.), and "Robustness analysis of floatingpoint programs by self-composition" (L. Chen et al.). In order to avoid routing livelock and guarantee multiple terminals' satisfaction, network modeling and scheduling are investigated in "Adaptive fault-tolerant routing in 2D mesh with cracky rectangular model" (Y. Yang et al.), "A bio-inspired QoSoriented handover model in heterogeneous wireless networks" (D. Tian et al.), and "An efficient multitask scheduling model for wireless sensor networks" (H. Yin et al.). After analyzing the frequencies of CVSS metrics, a vulnerability rating approach is proposed in the paper entitled "A software vulnerability rating approach based on the vulnerability database" (J. Luo et al.). Based on the comprehensive dictionary of Chinese words, "Reconstruction of uncertain historical evolution of the polysyllablization of Chinese lexis" (B. Qiu and J. Li) presents a new mapping approach from words to their occurrence times. 
The remaining 16 papers focus on the fields of formal modeling, checking, and verification.

A hybrid I/O automata model for an automated guided vehicle system is introduced in the paper entitled " $A$ case study on formal analysis of an automated guided vehicle system" (J. Zhang et al.). The hybrid automaton and Taylor approximation are studied in the paper entitled "Approximate equivalence of the hybrid automata with Taylor theory" (A. He et al.). Based on middle-model methodology, "Semantic consistency checking in building ontology from heterogeneous sources" (S. Yang et al.) proposes a model checking method to handle semantic consistency. Using the HOL theorem prover, the higher-order logic formalization of the function vector and the function matrix theories are proposed in the paper entitled "Formalization of function matrix theory in HOL" (Z. Shi et al.). Combining the extended modeling language CSP\# and linear temporal logic LTL, "Formal analysis of fairness for optimistic multiparty contract signing protocol" (by X. Li et al.) verifies the fairness of OMPCS protocols. Embedded system modeling and verification using Petri Nets are explored in the following papers: "Modeling, design, and implementation of a cloud workflow engine based on Aneka" (J. Zhou et al.), "Test purpose oriented I/O conformance test selection with colored Petri Nets" (J. Liu et al.), and "Modeling a heterogeneous embedded system in coloured petri Nets" ( $\mathrm{H}$. Zhang et al.). Based on the strand space model, "Formal modeling and analysis of fairness characterization of $E$ commerce protocols" (C. Zhang et al.) proposes a verification method for fairness performance. An approach exploiting the power of polynomial ring algebra is introduced in the paper entitled "Groebner bases based verification solution for SystemVerilog concurrent assertions" (N. Zhou et al.) to perform SystemVerilog assertion verification over digital circuit systems. The paper entitled "Functional verification of high performance adders in Coq" (Q. Wang et al.) proposes a systematic method to formalize and verify adders in formal proof assistant Coq. A technique for reducing the LTL formula is proposed in "Counterexample-preserving reduction for symbolic model checking" (W. Liu et al.). In the paper "Compositional abstraction refinement for componentbased systems" (L. Zhang et al.), the invariant strengthening and state partitioning techniques help to strengthen the abstraction and find counterexamples. The paper entitled "Terminal satisfiability in GSTE" (Y. Xu et al.) gives a sixtuple definition and presents a new algorithm for model checking terminal satisfiability. The paper entitled "Towards light-weight probabilistic model checking" (S. Konur) presents a methodology to facilitate probabilistic model checking for nonexperts. A machine closed theorem proof of TLA+ in the theorem proving system Coq is presented in "Formal proof of a machine closed theorem in Coq" ( $\mathrm{H}$. Wan et al.).

\section{Acknowledgments}

We sincerely thank the researchers who contributed to this special issue for their inspiring work, as well as the anonymous reviewers for their diligent assistance. In addition, we would like to express our appreciation for the editorial board members of this journal, who provided valuable help and support throughout the preparation of this special issue.

Guiming Luo

Xiaoyu Song

Xiaojing Yang

Krishnaiyan Thulasiraman 


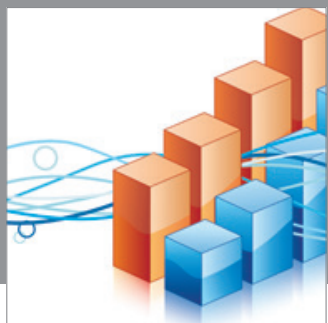

Advances in

Operations Research

mansans

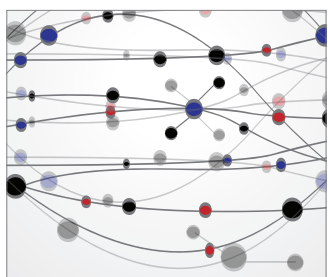

The Scientific World Journal
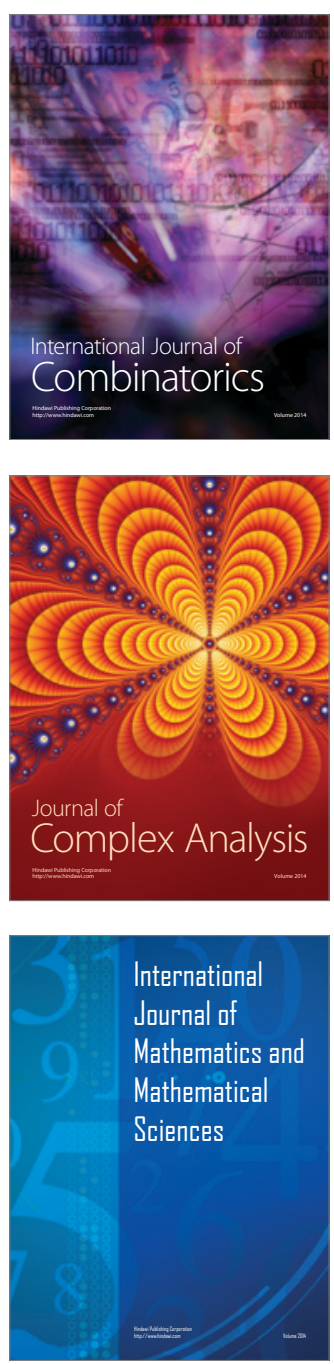
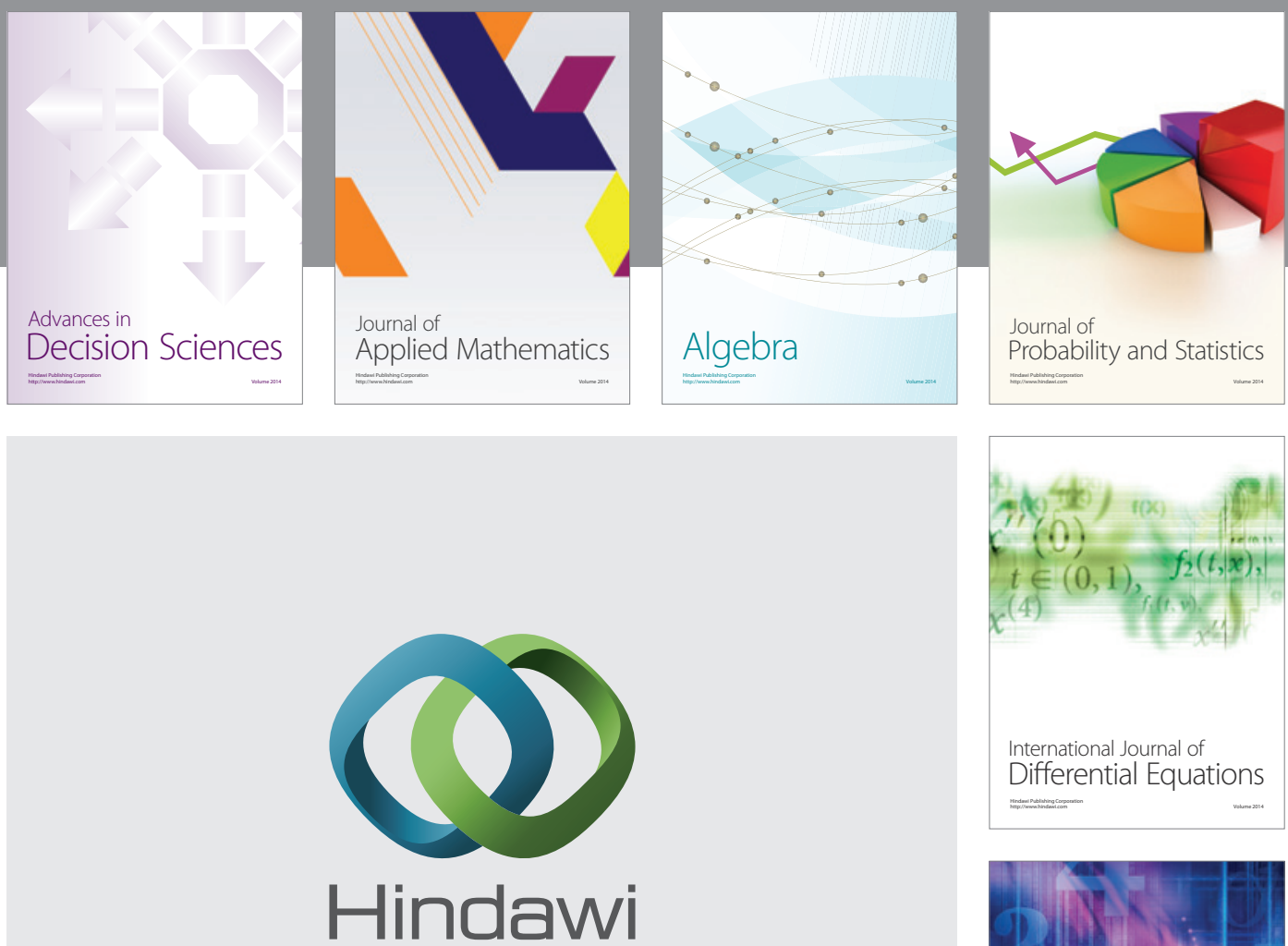

Submit your manuscripts at http://www.hindawi.com
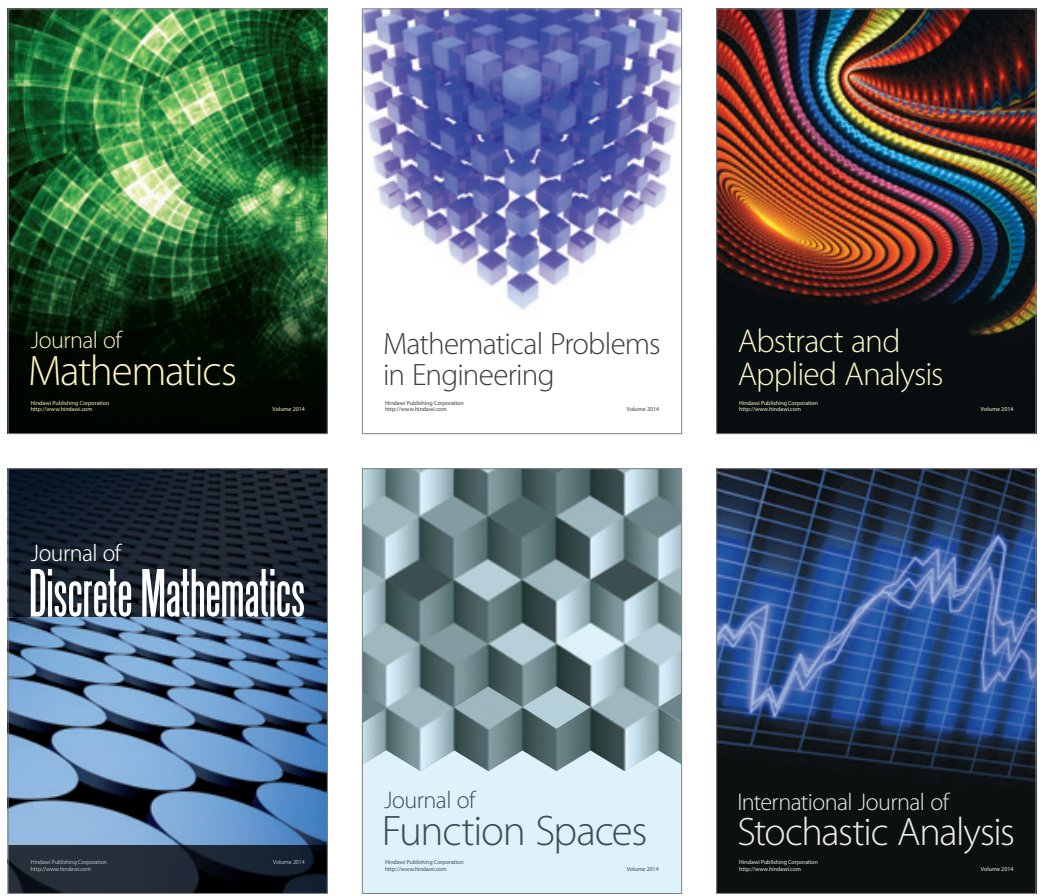

Journal of

Function Spaces

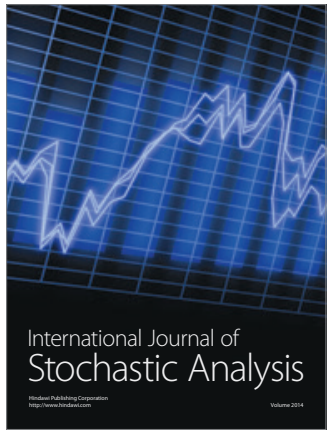

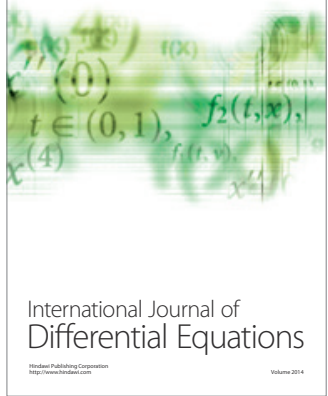
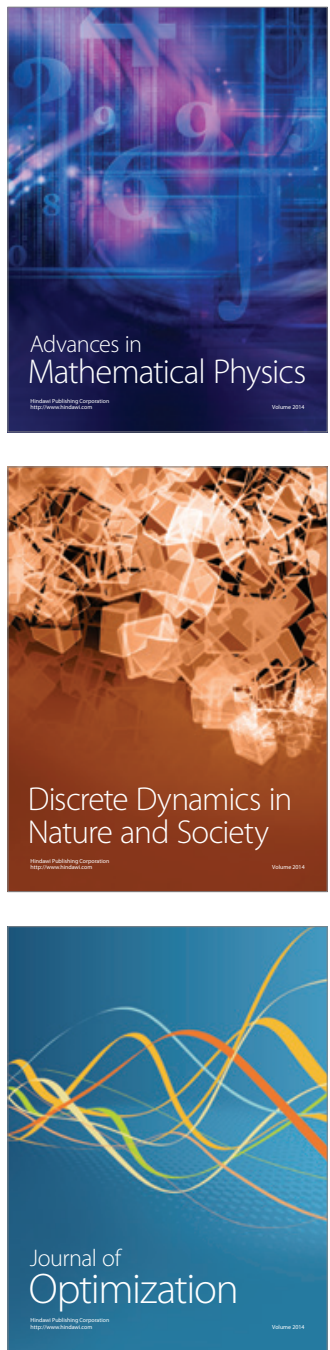